Algebraic 83 Geometric $\mathcal{T}$ opology

Volume 1 (2001) 369-380

Published: 3 June 2001

ATG

\title{
Topological geodesics and virtual rigidity
}

\author{
LOUIS FunAR \\ SidDhaRTHA GADGIL
}

\begin{abstract}
We introduce the notion of a topological geodesic in a 3-manifold. Under suitable hypotheses on the fundamental group, for instance word-hyperbolicity, topological geodesics are shown to have the useful properties of, and play the same role in several applications as, geodesics in negatively curved spaces. This permits us to obtain virtual rigidity results for 3-manifolds.
\end{abstract}

AMS Classification 57M10, 20F67; 57M50

Keywords Topological geodesic, word-hyperbolic group, residually finite, universal cover, virtual rigidity.

Geodesics in Riemannian manifolds with metrics of negative sectional curvature play an essential role in geometry. We show here that, in the case of 3 -dimensional manifolds, many crucial properties of geodesics follow from a purely topological characterization in terms of knotting. In particular, we prove two results concerning the virtual rigidity of 3-manifolds following the methods of Gabai [5].

We introduce the notion of a topological geodesic in a 3-manifold. We shall prove basic existence and uniqueness results for topological geodesics under suitable hypotheses on the fundamental group.

Suppose henceforth that $M$ is a closed 3-manifold with word-hyperbolic (or semi-hyperbolic) $\pi_{1}(M)$. We refer to the next section for the definition of the semi-hyperbolicity, following Alonso and Bridson. We adopt the convention here that finite groups are not semi-hyperbolic, hence all closed 3-manifolds we consider have infinite fundamental group unless the opposite is explicitly stated. In particular, the universal cover $\widetilde{M}$ of $M$ is homeomorphic to $\mathbb{R}^{3}$ (see $[2,9])$.

Definition 0.1 An embedded curve $\gamma$ in $M$ is a topological geodesic if a component $\widetilde{\gamma}$ of its inverse image in $\widetilde{M}$ is unknotted. 
Remark This is equivalent to saying that every component of its inverse image is unknotted. One observes that such a component is either a circle when $\gamma$ is torsion in $\pi_{1}(M)$ or a proper line in $\mathbb{R}^{3}$.

Under the hypothesis that $M$ is word-hyperbolic (or semi-hyperbolic) we have the following existence theorem.

Theorem (See theorem 1.2 and corollary 1.4) Let $M$ be an irreducible 3manifold with $\pi_{1}(M)$ word-hyperbolic (or semi-hyperbolic). Then every conjugacy class in $\pi_{1}(M)$ is represented by a topological geodesic.

If one further assumes that $\pi_{1}(M)$ is residually finite, we have an uniqueness result.

Theorem (See theorem 4.3) Let $M$ be an irreducible 3-manifold with $\pi_{1}(M)$ word-hyperbolic and residually finite. Suppose $c$ and $c^{\prime}$ are homotopic topological geodesics in $M$ representing a primitive class in $\pi_{1}(M)$ (i.e., not a multiple of any other class), then there exists a finite cover $M^{\prime}$ of $M$ such that $c$ and $c^{\prime}$ lift to isotopic curves in $M^{\prime}$.

In the case of a geodesic $\gamma$ in a Riemannian manifold, the exponential map is a surjection on a neighbourhood of $\gamma$, allowing one to construct a tubular neighbourhood. In the case of negative sectional curvature one can do more. Namely, a Hadamard-Cartan type argument allows one to construct thick tubular neighbourhoods when the injectivity radius is large. Thus, on passing to covers, we can ensure that we have thick tubes around a geodesic if the fundamental group is residually finite.

Again, we have an analogue of this property in the case of topological geodesics. This result is essentially present in the work of Gabai [5], who basically shows that thick tubes are present when topological geodesics exist and $\pi_{1}(M)$ is residually finite. Thus, the notion of topological geodesics, from the point of view of thick tubes, is implicit in Gabai's work.

Theorem (See theorem 2.1) Let $\gamma \in \pi_{1}(M)$ be a primitive element and let $k \in \mathbb{R}$. Then there is a geodesic $c \subset M$ and a finite cover $M^{\prime}$ of $M$ such that $c$ lifts to $M^{\prime}$ and there is an embedded solid torus $Q$ that contains $c$ and so that $d(\partial Q, c)$ is larger than $k$ and $Q-\operatorname{int}(N(c))=T^{2} \times[0,1]$. 
Remark Topological geodesics arise naturally while studying elliptic 3-manifolds. For instance, whether homotopy lens spaces are lens spaces is equivalent to the existence of (the analogue of) a topological geodesic. Further, lens spaces can be distinguished by considering the homotopy classes of topological geodesics in a given manifold.

We now turn to applications. A basic question in topology is to what extent the homotopy type of a manifold determines the manifold. For aspherical manifolds, in particular irreducible 3-manifolds with infinite fundamental group, conjecturally, pairs of homotopy equivalent manifolds are always homeomorphic.

One of the fundamental theorems in 3-manifold topology, due to Waldhausen, asserts that this is so for so-called Haken 3-manifolds. These include irreducible manifolds with non-trivial boundary. Following Gabai [5], we prove rigidity results by deleting solid tori and reducing to the case of manifolds with boundary. Partial results along these lines have also been obtained by Dubois [4] who simplified the previous proof by Gabai.

Our next result is that a large class of 3-manifolds are virtually rigid, i.e., pairs of homotopy equivalent manifolds have finite covers which are homeomorphic. Gabai [5] has shown this assuming residual finiteness and essentially the existence of topological geodesics.

Theorem 0.1 Suppose $M$ is a closed, irreducible 3-manifold with $\pi_{1}(M)$ infinite, residually finite and word-hyperbolic (or semi-hyperbolic). Then if $f: M \rightarrow N$ is a homotopy equivalence, there exist finite covers $M^{\prime}$ and $N^{\prime}$ of $M$ and $N$ and a lift $f^{\prime}: M^{\prime} \rightarrow N^{\prime}$ of $f$ which is homotopic to a homeomorphism.

Remark We only use word-hyperbolicity to show the tameness of certain covers of $M$, which also follows under some weaker hypotheses.

Our proof (which is already present in Gabai's paper) is a generalization of that of Gabai asserting the same when $N$ is hyperbolic. While this has subsequently been strengthened to showing rigidity for such manifolds (see [6] and [7]), the methods used are rather special to hyperbolic manifolds and are unlikely to generalise.

We also prove, under the same hypothesis, another rigidity result of a complementary nature. This uses both the existence and uniqueness of topological geodesics. 
Theorem (See theorem 5.1) Let $M$ be irreducible and with word-hyperbolic fundamental group. If $f: M \rightarrow M$ is a homeomorphism homotopic to the identity then there is a finite cover $M^{\prime}$ of $M$ and a lift $f^{\prime}: M^{\prime} \rightarrow M^{\prime}$ of $f$ such that $f^{\prime}$ is isotopic to the identity.

Further applications of topological geodesics will be studied elsewhere.

Acknowledgements We would like to thank Yair Minsky, Darren Long and David Gabai for helpful comments and conversations.

\section{Definition and Existence}

We assume henceforth that $M$ is a closed, irreducible 3-manifold with $\pi_{1}(M)$ word-hyperbolic, residually finite (and infinite). We shall generalize this to the semi-hyperbolic case, but we begin with the word-hyperbolic case which is easier. We make (as in the introduction) the following definition.

Definition 1.1 An embedded curve $\gamma$ in $M$ is a topological geodesic if a component $\widetilde{\gamma}$ of its inverse image in $\widetilde{M}$ is unknotted.

The existence of geodesics is based on the following lemma.

Lemma 1.1 For $\gamma \in \pi_{1}(M)$, let $M_{\gamma}=\widetilde{M} /\langle g\rangle$ be the quotient of $\widetilde{M}$ by the the group of deck transformations generated by $\gamma$. Then $\widetilde{M} /\langle g\rangle=S^{1} \times \mathbb{R}^{2}$.

Proof As $\pi_{1}(M)$ is word-hyperbolic, the universal cover $\widetilde{M}$ has a compactification to $B^{3}$, and the action by deck transformations extends to $B^{3}$. The action of $\gamma$ has two fixed points $p$ and $q$, and $\gamma$ acts properly discontinuously on $B^{3}-\{p, q\}$ with quotient $D^{2} \times S^{1}$. The result follows as $M_{\gamma}$ is the interior of this manifold.

Theorem 1.2 Let $M$ be an irreducible 3-manifold with word-hyperbolic $\pi_{1}(M)$. Then given $\gamma \in \pi_{1}(M)$, there is a topological geodesic $c$ that represents $\gamma$.

Proof We simply take the image in $M$ of a curve $c$ in $M_{\gamma}$ that is a core of the solid torus constructed above. It is easy to ensure that the image is embedded. 
Remark The above theorem shows that there exists a topological geodesic representing every element in the fundamental group, rather than each conjugacy class of elements, which is the case for geodesics in a Riemannian manifold with negative sectional curvature. But given a geodesic representing an element, there is an obvious construction of a geodesic representing any conjugate element. So the real issue is that the topological geodesic representing a conjugacy class should be unique.

Next, we weaken the hypothesis on $\pi_{1}(M)$. For the sake of completeness we outline the definition of a semi-hyperbolic group below (see [1]).

Definition 1.2 For a metric space $(X, d)$ set $P(X)$ for the set of eventually constant maps $p: \mathbb{Z}_{+} \rightarrow X$, thought as finite discrete paths in $X$. For $p \in$ $P(X)$ one denotes by $T_{p}$ the smallest integer at which $p$ becomes constant. A bicombing of $X$ consists of a choice of a (combing) path $s_{(x, y)} \in P(X)$ joining the points $x$ and $y$ of $X$, for all $x, y \in X$.

- The bicombing $s$ is quasi-geodesic if there exist constants $\lambda, \varepsilon$ such that $\left.\left.s_{(x, y)}\right|_{\left[0, T_{s}(x, y)\right.}\right]$ is a $(\lambda, \varepsilon)$-quasi-geodesic, for all $x, y \in X$. This means that

$$
d\left(s_{(x, y)}(t), s_{(x, y)}\left(t^{\prime}\right)\right) \leq \lambda\left|t-t^{\prime}\right|+\varepsilon,
$$

is fulfilled for all $x, y \in X, t, t^{\prime} \in \mathbb{Z}_{+}$, thus the combing paths are uniformly closed to geodesics.

- The bicombing $s$ is bounded if there exist constants $k_{1} \geq 1, k_{2} \geq 0$ such that for all $x, y, x^{\prime}, y^{\prime} \in X, t \in \mathbb{Z}_{+}$one has

$$
d\left(s_{(x, y)}(t), s_{\left(x^{\prime}, y^{\prime}\right)}(t)\right) \leq k_{1} \max \left\{d\left(x, x^{\prime}\right), d\left(y, y^{\prime}\right)\right\}+k_{2} .
$$

- The metric space $X$, acted upon isometrically by the discrete group $\Gamma$, is said to be a semi-hyperbolic $\Gamma$-metric space if it admits a bounded quasigeodesic bicombing $s$ which is $\Gamma$-equivariant i.e. $\gamma s_{(x, y)}(t)=s_{(\gamma x, \gamma y)}(t)$ holds for all $x, y \in X, \gamma \in \Gamma, t \in \mathbb{Z}_{+}$.

- The (finitely generated) group $\Gamma$ is semi-hyperbolic if $\Gamma$ with the word metric (associated to some system of generators) is a semi-hyperbolic $\Gamma$-metric space, with respect to the action by left multiplication.

The semi-hyperbolicity is independent on the system of generators. It is known that hyperbolic groups and biautomatic groups are semi-hyperbolic.

Lemma 1.3 Assume $\gamma$ is an element of infinite order and $\pi_{1}(M)$ is a $C A T(0)$ group (i.e. it acts properly discontinuously and co-compactly by isometries on a geodesic $C A T(0)$ metric space), or more generally a semi-hyperbolic group. Then the conclusion of lemma 1.1 holds true. 
Proof Any infinite cyclic subgroup $\mathbb{Z} \subset \pi_{1}(M)$ yields a geodesic line in $X$ by the Flat Torus Theorem (see $[1,13])$ and so it is a quasi-convex subgroup (see [1], Thm.9.11 for details). The main theorem of [9] implies that $\widetilde{M} /\langle\gamma\rangle$ is a missing boundary manifold, hence the interior of a solid torus. In the semi-hyperbolic case an infinite cyclic group lies in the center of its centralizer (which is a finite extension of the infinite cyclic group) and the latter is both semi-hyperbolic and a quasi-convex subgroup of $\pi_{1}(M)$.

Corollary 1.4 In the hypotheses of theorem 1.2 we may replace word-hyperbolic by semi-hyperbolic.

Thus, we may henceforth weaken our hypothesis on $\pi_{1}(M)$ to require only semi-hyperbolicity in place of word hyperbolicity.

Using some results of M.Bridson one can extend the previous lemma to nil and sol 3-manifolds, thus holding true for all fundamental groups of geometric 3-manifolds. Notice that M.Kapovich has announced that atoroidal CAT(0) 3-manifold groups are word hyperbolic, which improves on a previous theorem of L.Mosher.

\section{Thick tubes}

In this section, we show that a geodesic $c$ corresponding to a primitive element of $\pi_{1}(M)$ has a thick tube around it in a finite cover, i.e., a solid torus $Q$ that contains $c$ and so that $d(\partial Q, c)$ is large and $T-\operatorname{int}(N(c))=T^{2} \times[0,1]$. The precise statement is below. Here, and henceforth, we fix a metric on $M$ and use the pull-back metric on all its covers

The construction is based on the fact due to Darren Long (see [8]) that in word-hyperbolic, or more generally atoroidal, groups, maximal cyclic groups are separable.

Theorem (D. Long) Let $G$ be a residually finite group, $\gamma \in G$ an element that generates a maximal abelian subgroup, and $S$ a finite set disjoint from $\langle\gamma\rangle$. Then there exists a subgroup $H \subset G$ with finite index such that $\gamma \in H$ but $S \cap H=\emptyset$.

We construct a thick tube in the cover $\widetilde{M} /\langle\gamma\rangle=S^{1} \times \mathbb{R}^{2}$. This embeds in a cover where all other small elements are separated from such a group. In this cover we have a short curve (geodesic) in one homotopy class, but all closed 
curves that are not homotopic to a power of this are long. The precise statement and proof are below.

Theorem 2.1 Let $\gamma \in \pi_{1}(M)$ be a primitive element and let $k \in \mathbb{R}$. Then there is a geodesic $c \subset M$ and a finite cover $M^{\prime}$ of $M$ such that $c$ lifts to $M^{\prime}$ and there is an embedded solid torus $Q$ that contains $c$ and so that $d(\partial Q, c)>k$ and $Q-\operatorname{int}(N(c))=T^{2} \times[0,1]$.

Proof Consider the cover $M_{\gamma}=\widetilde{M} /\langle g\rangle$ of $M$ and pick base-points $p$ of $M$ and $p^{\prime}$ of $M_{\gamma}$. As $M_{\gamma}$ is homeomorphic to $S^{1} \times R^{2}$, there is a curve $c$ and a solid torus $Q$ embedded in $M_{\gamma}$, with $c$ based at $p^{\prime}$, so that $c$ and $Q$ are as required. We shall find a finite cover $M^{\prime}$ of $M$ which is covered by $M_{\gamma}$ so that the image of $Q$ embeds in $M^{\prime}$.

As $Q$ is compact, there is a finite collection $X$ of inverse images of $p$ in $M_{\gamma}$ such that if each of these map to distinct points in an intermediate cover $M^{\prime}$ between $M_{\gamma}$ and $M$, then $Q$ embeds in this cover. Choose paths joining $p^{\prime}$ to each point in $X$ and let $S^{\prime}$ be the set of elements in $\pi_{1}(M)$ that are represented by the images of these paths. Let $S=S^{\prime} \cup\left\{x y^{-1}: x, y \in S\right\}$.

By the above theorem, there exists a subgroup $H$ separating $\gamma$ from $S$. Let $M^{\prime}$ be the corresponding cover. It is easy to see that the elements of $X$ map to different points in $M^{\prime}$, and hence $Q$ embeds in $M^{\prime}$.

Definition 2.1 A thick tube around a geodesic $c$ is an embedded solid torus $Q$ that contains $c$ such that $Q-\operatorname{int}(N(c))=T^{2} \times[0,1]$.

Observe that the components of the inverse image of a thick tube around a geodesic in $\widetilde{M}$ are unknotted.

\section{Virtual rigidity}

Gabai's proof of virtual rigidity (see [5]) now generalizes. Briefly, if there are knots $K$ and $K^{\prime}$ in $M$ and $N$ whose complements are irreducible and so that, possibly after changing $f$ by a homotopy, $f(N(K)) \subset N\left(K^{\prime}\right)$ and $f(M-$ $\operatorname{int}(N(K))) \subset N-\operatorname{int}\left(N\left(K^{\prime}\right)\right)$, then we can apply Waldhausen's result to the knot exteriors. Gabai's proof proceeds by showing that given a geodesic $\gamma$ in $M$ (which plays the role of $K$ ) with a thick tube around it, the image of this contains a solid torus $W$ (which plays the role of $N\left(K^{\prime}\right)$ ), so that $f$ can be 
deformed to a map that takes $M-\operatorname{int}(N(\gamma))$ to $N-\operatorname{int}(W)$. Waldhausen's theorem now shows rigidity.

As $f$ is a homotopy equivalence between compact manifolds, there exists $C$ such that $d(x, y) \geq C \Longrightarrow f(x) \neq f(y)$. By the methods of the previous section, given $C \in \mathbb{R}$ there is a finite cover $M^{\prime}$ of $M$, a geodesic $\gamma \subset M^{\prime}$ and solid tori $V_{i}, 1 \leq i \leq 4$ such that $d\left(\gamma, \partial V_{1}\right)>C$ and $d\left(\partial V_{i}, \partial V_{i+1}\right)>C, 1 \leq i \leq 3$. Let $S_{i}=\partial V_{i}$. Let $V_{0}=\gamma$. Replace $M$ by $M^{\prime}$ and $N$ by its cover with the same fundamental group as $M^{\prime}$ (under the identification $\pi_{1}(M) \stackrel{f_{*}}{=} \pi_{1}(N)$.

The rest of the proof is exactly the same as that of Gabai (hyperbolicity is not used beyond this stage - only that the tori are far apart and topologically of a standard form). We outline below the main steps.

Let $g: N \rightarrow M$ be the homotopy inverse of $f$. Let $K=f\left(S_{2}\right)$ and $J=$ $N(K) \cup$ (components of $N-K$ disjoint from $\left.f\left(S_{1}\right) \cup f\left(S_{3}\right)\right)$. Then $\partial J$ has two components, one of which bounds a region disjoint from $J$ containing $f\left(S_{1}\right)$ and the other bounds a region disjoint from $J$ containing $f\left(S_{3}\right)$. Further, $J$ is irreducible and $[K]$ generates $H_{2}(J)=\mathbb{Z}$. All this follows from the fact that $d(x, y) \geq C \Longrightarrow f(x) \neq f(y)$.

Next, $J$ contains a homologically non-trivial torus which bounds in $N$ a solid torus $W$ containing $f(\gamma)$. This is constructed using the fact that the Thurston norm equals the singular norm, and that we have a singular torus $T$. Further, $g: T \rightarrow N-\operatorname{int}(W)$ and the inclusion $T \rightarrow N-\operatorname{int}(W)$ induce injections between the fundamental groups.

Now we can deform $f$ and $g$ so that they restrict to give a homotopy equivalence between $M-V_{2}$ and $N-W$. Waldhausen's theorem now gives the result.

\section{Uniqueness}

Lemma 4.1 (Engulfing lemma) Any curve $d$ in $M$ homotopic to a geodesic $c$ is contained in a tube around the geodesic $c$ in some finite cover.

Proof As $c$ and $d$ are homotopic, there exists an annulus $A$ with boundary components $c$ and $d$. On passing to a cover with a sufficiently thick tube $Q$ around $c$, the annulus $A$ is contained in the tube $Q$. Hence $d$ has a lift that is contained in $Q$.

Lemma 4.2 (Core lemma) If $c$ and $c^{\prime}$ are homotopic geodesics and $c^{\prime}$ is contained in a thick tube $Q$ around $c$, then $c^{\prime}$ is isotopic to $c$. 
Proof Consider $\pi_{1}\left(Q-c^{\prime}\right)$. The inverse image of the solid torus $Q$ in $\widetilde{M}$ contains an unknotted cylinder $D$ that contains a unique component $C^{\prime}$ of the inverse image of $c^{\prime}$. The group $\pi_{1}\left(D-C^{\prime}\right)=\pi_{1}\left(\widetilde{M}-C^{\prime}\right)$ is the kernel of the map $\pi_{1}\left(Q-c^{\prime}\right) \rightarrow \mathbb{Z}$ that maps the longitude of the torus $\partial Q$ (hence those of $c$ and $c^{\prime}$ ), to 1 and the meridian to 0 . As $C^{\prime}$ is an unknot (because $c^{\prime}$ is a geodesic) this kernel is $\mathbb{Z}$, hence $\pi_{1}\left(Q-c^{\prime}\right)=\mathbb{Z}^{2}$. This implies that $c^{\prime}$ is isotopic to $c$.

Theorem 4.3 Let $M$ be an irreducible 3-manifold with $\pi_{1}(M)$ word-hyperbolic (or semi-hyperbolic) and residually finite. Suppose $c$ and $c^{\prime}$ are homotopic topological geodesics in $M$ representing a primitive class in $\pi_{1}(M)$. Then there exists a finite cover $M^{\prime}$ of $M$ such that $c$ and $c^{\prime}$ lift to isotopic curves in $M^{\prime}$.

Proof As $c$ and $c^{\prime}$ are homotopic, there is an annulus $A$ bounding $c$ and $c^{\prime}$. By passing to a cover $M^{\prime}$ with a sufficiently thick tube $Q$ around $c$, we may ensure that $A$ is contained in $Q$. The Core lemma now implies that $c$ and $c^{\prime}$ are isotopic.

\section{Homotopy versus isotopy}

It is known that for an irreducible manifold homotopic self-homeomorphisms are isotopic provided that the manifold is hyperbolic (a consequence of Gabai's rigidity [6]) or Seifert fibered (by the Scott theorem) or lens spaces.

Theorem 5.1 Let $M$ be irreducible and with word-hyperbolic fundamental group. If $f: M \rightarrow M$ is a homeomorphism homotopic to the identity then there is a finite cover $M^{\prime}$ of $M$ and a lift $f^{\prime}: M^{\prime} \rightarrow M^{\prime}$ of $f$ such that $f^{\prime}$ is isotopic to the identity.

Proof Let $\gamma$ be a topological geodesic corresponding to a primitive class in $\pi_{1}(M)$. Then the geodesics $f(\gamma)$ and $\gamma$ are homotopic hence there exists a finite cover $M^{\prime}$ such that $f(\gamma)$ and $\gamma$ lift to isotopic curves. Since $f$ is homotopic to identity there is no obstruction in lifting it to a homotopy equivalence $f^{\prime}$ of the finite covering $M^{\prime}$. One can further assume (by means of some isotopy on $M^{\prime}$ ) that $f\left(\gamma^{\prime}\right)=\gamma^{\prime}$ (pointwise).

The proof of the core lemma shows that $M^{\prime}-\gamma^{\prime}$ is atoroidal hence hyperbolic (since Haken). Furthermore the restriction $\left.f^{\prime}\right|_{M^{\prime}-\gamma^{\prime}}$ is a homeomorphism. 
Therefore $\left.f^{\prime}\right|_{M^{\prime}-\gamma^{\prime}}$ is homotopic to an isometry of $M^{\prime}-\gamma^{\prime}$ (by Mostow rigidity) and hence isotopic to an isometry (by Waldhausen's theorem).

Let $j$ be this isometry. Since the isometry group of a hyperbolic manifold of finite volume is finite it follows that $j$ is of finite order. Further $j$ has an extension $g$ (by identity) to all of $M^{\prime}$, by asking $g$ to keep pointwise $\gamma^{\prime}$. In particular $g$ is a periodic homeomorphism of $M^{\prime}$.

Let consider the lift $h$ of $g$ to the universal covering $\widetilde{M}=\mathbf{R}^{3}$ (which is also the universal covering of $M^{\prime}$ ). The action of $h$ extends continuously to the compactification (over the boundary sphere) to a homeomorphism of the ball $B^{3}$. The action by deck transformations extends to one by homeomorphisms of the compactification obtained by adding the boundary of the group $\pi_{1}(M)$, because this is word-hyperbolic. In our case the boundary is the sphere at infinity.

But $f$ is homotopic to identity, hence the action induced on the boundary is trivial. This shows that $h$ is the identity on the boundary sphere.

Lemma 5.2 A periodic homeomorphism of $B^{3}$ which restricts to identity on the boundary is the identity.

Proof We will use a theorem of Newman ([10]) improved by Smith (see [12]) in its variant stated in ([3],Thm.9.5, p.157). It states that a compact Lie group acting effectively on a connected topological manifold has a nowhere dense fixed point set.

One considers the finite cyclic group action induced by our periodic homeomorphism on the ball $B^{3}$. This action extends to the sphere $S^{3}$ by the identity outside the upper hemisphere. Then the fixed point set contains a 3 -ball and the previous result shows that the action cannot be effective, and hence $h$ must be the identity map.

In particular $f^{\prime}$ is isotopic to the identity.

\section{Concluding remarks}

While we have assumed word-hyperbolicity (or semi-hyperbolicity) for the sake of definiteness, we actually need much less. Assuming $\pi_{1}(M)$ is atoroidal, we also need everywhere that $\widetilde{M}=\mathbb{R}^{3}$, or equivalently that $\widetilde{M}^{3}$ is tame (i.e, is the 
interior of a compact manifold). Existence and uniqueness use the tameness of $M_{\gamma}$. Finally, virtual rigidity needs tameness for some $M_{\gamma}$.

There are contractible 3-manifolds, namely Whitehead manifolds, different from $\mathbb{R}^{3}$. There are also 3-manifolds different from $S^{1} \times \mathbb{R}^{2}$ that have fundamental group $\mathbb{Z}$ and universal cover $\mathbb{R}^{3}$ (see, for example, [11]). Conjecturally these manifolds do not admit free co-compact actions, but so far this is known only under additional assumptions on the group (for example word-hyperbolicity).

One can generalize the notion of topological geodesics to include knots having a lift in $\mathbb{R}^{3}$ whose fundamental group is free (or even residually nilpotent following [4]). Although weak versions of the uniqueness could be proved in this context, the obstruction in deriving rigidity results is the existence. The existence of at least one topological geodesic (homotopically nontrivial) appears to be as difficult in this more general framework as in the present case.

An even weaker notion of a topological geodesic is one whose lift to the universal cover is not a satellite knot. In the presence of topological geodesics, the proof of the Core lemma shows that any curve that is not a topological geodesic lifts to a finite cover where it is a satellite knot, and hence is also not a topological geodesic in this weaker sense.

\section{References}

[1] J.M.Alonso and M.R.Bridson, Semihyperbolic groups, Proc. London Math. Soc. (3) $\mathbf{7 0}$ (1995), 56-114.

[2] M.Bestvina and G. Mess, The boundary of negatively curved groups, J. Amer. Math. Soc. 4(1991), 469-481.

[3] G.E.Bredon, Introduction to compact transformation groups, Pure Appl. Math., Academic Press, 1972.

[4] J. Dubois, Noeuds Fox-résiduellement nilpotents et rigidité virtuelle des variétés hyperboliques de dimension 3, Ann.Inst.Fourier, Grenoble, 48(1998), 535-551.

[5] D. Gabai, Homotopy hyperbolic 3-manifolds are virtually hyperbolic, J. Amer. Math. Soc. 7(1994), 193-198.

[6] D.Gabai, On the geometric and topological rigidity of hyperbolic 3-manifolds, J. Amer. Math. Soc. 10 (1997), no. 1, 37-74.

[7] D.Gabai, G.R.Meyerhoff and N.Thurston, Homotopy hyperbolic 3-manifolds are hyperbolic, Annals of Math.(to appear).

[8] D.Long, Immersions and embeddings of totally geodesic surfaces, Bull. London Math. Soc. 19 (1987), no. 5, 481-484. 
[9] M.L.Mihalik, Compactifying coverings of 3-manifolds, Comment.Math.Helvetici 71(1996), 362-372.

[10] M.H.A.Newman, A theorem on periodic transformations of spaces, Quart.J. Math. Oxford 2(1931), 1-8.

[11] G.P.Scott and T.Tucker, Some examples of exotic noncompact 3-manifolds, Quart. J. Math. Oxford Ser. (2) 40 (1989), no. 160, 481-499.

[12] P.A.Smith, Transformations of finite period, III: Newman's theorem, Ann. of Math. 42(1941), 446-458.

[13] R.L. Volkovyskii, Plane geodesically complete subsets in spaces of nonpositive curvature Algebra i Analiz 6 (1994), no. 4, 90-100; translation in St. Petersburg Math. J. 6 (1995), no. 4, 781-789.

Institut Fourier BP74, UMR 5582, Universite de Grenoble I

38402 Saint-Martin-d'Heres cedex, France

and

Department of Mathematics, SUNY at Stony Brook

Stony Brook, NY 11794, USA

Email: funar@fourier.ujf-grenoble.fr and gadgil@math.sunysb.edu

Received: 4 April 2001

Algebraic \& $\mathcal{G}$ eometric Topology, Volume 1 (2001) 